\title{
Lymphopenia and Radiotherapy for Geriatric Cancer Patients in the Reality of COVID-19
}

(1) Fatma SERT

Department of Radiation Oncology, Ege University, Faculty of Medicine, İmir-Turkey

\section{Dear Editor,}

Regarding COVID-19, which is now accepted as a pandemic, many questions have been generated about how to manage and postpone the treatment of cancer patients, especially for the patients in geriatric age. It was determined that the incidence of lymphopenia increased in COVID-19 and there was a significant relationship between lymphopenia, age and mortality. This can be accepted as an unsolved problem in how to maintain radiotherapy that cause lymphopenia. In radiation oncology, which is comprised of the majority of geriatric patients, treatment decisions, the application of the treatment and the continuation of the treatment are particularly important. It is essential not to ignore this geriatric population in the COVID-19 pandemic, which concerns the whole society. This letter was written for taking attention of professional staff that are responsible for geriatric cancer patients diagnosed with COVID-19.

A virus-related infection called 2019 novel coronavirus disease (COVID-19), which is now accepted as a pandemic, has negatively affected daily life in all countries, and has become a significant cause of death globally.[1] After detection of an atypical viral pneumonia case cluster in late 2019, a new coronavirus was isolated from these cases in Wuhan, China in January 2020 which was named severe acute respiratory syndrome coronavirus 2 (SARS-CoV-2) and was reported to cause a serious pneumonia.[1] Every single country faced to fight with COVID-19 to prevent its spread as if they were all in a real war.

In the light of the current data, although $81 \%$ of patients with COVID-19 develop a milder form of dis- ease, $14 \%$ have a severe illness requiring hospitalization and additional oxygen, and the remaining 5\% develop respiratory failure, septic shock, and/or multi-organ dysfunction. Again, the current data show that COVID-19 case death rates are approximately $2 \%$, and it rises to $15 \%$ in patients aged 80 and over.[1] Cancer patients have a higher risk of developing COVID-19 and a higher risk of mortality as well.[2] Immune suppression that arises from the tumor and its treatment is also a plausible contributory factor [1], which suggests that oncologists should carefully weigh the benefits of treatment offered to their patients against the risks posed by COVID- 19 .

Radiotherapy (RT) is an essential part of cancer treatment. The bone marrow suppression effect of RT has been shown, and this includes depletion of circulating lymphocytes.[3,4] Pre-treatment lymphopenia, which has been identified as a poor prognostic factor in many malignancies, is probably a reflection of tumor-induced immune suppression. [5] Low lymphocyte count was reported to be associated with increased disease severity in COVID-19, and patients who died of COVID-19 had significantly lower lymphocyte counts than survivors. As a result, this RT-induced lymphopenia is considered to be an important prognostic laboratory marker both for infected COVID-19 and mortality due to COVID-19.[5] Deferring treatment in patients with good prognostic tumors with a low risk of progression, such as lowand intermediate-risk prostate cancer, has also been recommended.[6] Since, shown a positive correlation between age and lymphopenia rate exists, we should evaluate our geriatric cancer patients before RT start. 
Additionally, limited access to food supply and fear of contacting the disease in a public area may alter their nutrition and depress their immunity. Lymphopenia can be a real problem for the geriatric population due to this depressed immunity.[7] Rui et al. published their clinical investigation in geriatric patients with COVID-19.[8] All the evaluated patients had lymphopenia, and they concluded that lymphopenia could be an unfavorable factor for mortality due to COVID-19 pneumonia. Similarly, An et al. reported that lymphopenia frequently occurs in COVID-19 patients, manifested as the decrease of total T cells, CD4+T cells and $\mathrm{CD} 8+\mathrm{T}$ cells in the peripheral blood; with the degree of decline related to the severity of the disease. Compared with patients with mild symptoms, a higher proportion of severe or deceased patients had lymphopenia, which is similar to the observation during SARS-CoV infection. [9]

What about RT for geriatric cancer patients? We do not have chance to leave these populations with their unexpected faith. We should try to look at the new COVID-19 pandemic process from the geriatric cancer patients' window. We could not delay RT at least for palliative necessities and definitive purposes. On the other hand, we all agree that it is necessary to consider benefit versus the risk in using RT to geriatric cancer patients in the reality of COVID-19 pandemia. However, we should not fear to use palliative RT when there is a certain indication. Additionally, we should learn not only the reasons for lymphopenia but also the management of it.

\section{References}

1. Guan WJ, Ni ZY, Hu Y, Liang WH, Ou CQ, He JX, et al. Clinical Characteristics of Coronavirus Disease 2019 in China. N Engl J Med. 2020;382(18):1708-20.

2. Xia Y, Jin R, Zhao J, Li W, Shen H. Risk of COVID-19 for patients with cancer. Lancet Oncol 2020;21(4):180.

3. Formenti SC, Demaria S. Combining radiotherapy and cancer immunotherapy: a paradigm shift. J Natl Cancer Inst 2013;105(4):256-65.

4. Sert F, Yalman D, Özkök S. Lymphopaenia and accidental splenic doses: Do they have any prognostic value for locally advanced gastric cancer patients treated with radiochemotherapy? Contemp Oncol (Pozn) 2019;23(4):226-33.

5. Zhao J, Huang W, Wu Y, Luo Y, Wu B, Cheng J, et al. Prognostic role of pretreatment blood lymphocyte count in patients with solid tumors: a systematic review and meta-analysis. Cancer Cell Int 2020;20:15.

6. Zaorsky NG, Yu JB, McBride SM, Dess RT, Jackson WC, Mahal BA, et al. Prostate Cancer Radiotherapy Recommendations in Response to COVID-19. Adv Radiat Oncol 2020;5(4):659-65.

7. Nguyen NP, Vinh-Hung V, Baumert B, Zamagni A, Arenas M, Motta M, et al. Older Cancer Patients during the COVID-19 Epidemic: Practice Proposal of the International Geriatric Radiotherapy Group. Cancers (Basel) 2020;12(5):1287.

8. Rui L, Sirui L, Xuebei D, Xujun Y, Yanggan W. Clinical observations in very elderly patients with COVID-19 in Wuhan. Geriatr Gerontol Int 2020;20(7):709-14.

9. An PJ, Zhu YZ, Yang LP. Biochemical indicators of coronavirus disease 2019 exacerbation and the clinical implications. Pharmacol Res 2020 May 23 (ePub). 